\title{
Gravidez na adolescência e violência doméstica no contexto da atenção primária à saúde
}

\author{
Teenage pregnancy and domestic violence in the context of primary health care \\ El embarazo en la adolescencia y la violencia doméstica en el contexto de la atención primaria de salud
}

\author{
Camilla Moura Aguiar1® ${ }^{\circledR}$, Kilma Wanderley Lopes Gomes²® \\ ${ }^{1}$ Fundação Oswaldo Cruz, FIOCRUZ, Rio de Janeiro - RJ, Brasil \\ ${ }^{2}$ Universidade de Fortaleza, UNIFOR, Fortaleza - CE, Brasil
}

\section{Resumo}

Introdução: A gravidez na adolescência tem ainda alta incidência no Brasil, mesmo após grande redução nas últimas décadas, e pode estar relacionada a situações de vulnerabilidade social e de violência doméstica. A Estratégia de Saúde da Família (ESF) é fundamental na atenção à saúde do adolescente, entretanto tem se mostrado pouco atuante no enfrentamento das violências nessa faixa etária. Objetivo:Este estudo objetivou descrever o perfil socioeconômico e identificar características materno-fetais e situações de vulnerabilidade social das jovens com histórico de gravidez na adolescência, analisando possíveis associações com a ocorrência de violência doméstica. Metodologia: Trata-se de estudo transversal, com entrevista de 100 adolescentes entre 13 e 19 anos com histórico de gravidez em 2018 de bairro pobre de Fortaleza - CE. Foi aplicado questionário com 57 questões e as variáveis analisadas foram as relacionadas ao perfil sociodemográfico, ao seguimento do pré-natal, ao nascimento da criança e à exposição à violência doméstica durante a gestação. Foram realizados teste qui-quadrado $\left(\chi^{2}\right)$ e Odds Ratio $(O R)$ na análise. Resultados: $A$ idade média das entrevistadas foi 17,5 anos $(\mathrm{dp}=1,65)$. A renda familiar média foi 1,18 saláriomínimo ( $\mathrm{dp}=0,83$ ), $91 \%$ eram negras ou pardas, $57 \%$ estavam em união consensual e $18 \%$ tinham emprego. Com relação à escolaridade, $71 \%$ haviam interrompido os estudos, sendo que $46,5 \%$ delas o fez antes da primeira gravidez e $35,2 \%$ pararam de estudar depois de engravidar. No seguimento de pré-natal, $96,3 \%$ realizaram pelo menos uma consulta, $62,1 \%$ iniciaram no primeiro trimestre e $69,1 \%$ realizaram 6 ou mais consultas. Dentre as crianças, $7,6 \%$ e $6,4 \%$ nasceram prematuras e com baixo peso respectivamente. A porcentagem de violência doméstica foi $26 \%$ e o principal agressor foi o companheiro. Sofrer violência doméstica teve associação estatisticamente significativa com baixa escolaridade (OR 4,06; IC95\% 1,27-12,97), menor idade materna (OR 4,2; IC95\% 1,4312,32) e "história de internação de recém-nascido" (OR 3,83; IC95\% 1,34-10,95). Conclusão: As mães adolescentes estavam inseridas em contexto de vulnerabilidade social e parte considerável foi vítima de violência durante a gestação, situação associada à baixa escolaridade e menor idade da adolescente. As consequências negativas para saúde do recém-nascido foram mais frequentes em situações de violência. Notou-se que as adolescentes tiveram boa frequência no pré-natal e isso pode ter influenciado positivamente os desfechos obstétricos e neonatais. A gestação é, muitas vezes, o primeiro contato dessa jovem com o serviço de saúde e acesso à assistência pré-natal na ESF pode reduzir complicações materno-infantis e auxiliar na identificação da violência doméstica.

Palavras-chave: Gravidez na adolescência, Atenção Primária à Saúde, Gênero e Saúde, Violência Doméstica, Vulnerabilidade Social.

Como citar: Aguiar CM, Gomes KWL. Gravidez na adolescência e violência doméstica no contexto da atenção primária à saúde. Rev Bras Med Fam Comunidade. 2021;16(43):2401. https://doi.org/10.5712/rbmfc16(43)2401

\author{
Autor correspondente: \\ Camilla Moura Aguiar. \\ E-mail: camillacami@hotmail.com \\ Fonte de financiamento: \\ não se aplica. \\ Parecer CEP: \\ CAAE: 85882418.6.0000.5045 \\ Procedência: \\ não encomendado. \\ Avaliação por pares: \\ externa. \\ Recebido em: 15/02/2020. \\ Aprovado em: 31/03/2021.
}




\begin{abstract}
Introduction: Teenage pregnancy still have a high incidence in Brazil, even after a great reduction in the last decades, and may be related to situations of social vulnerability and domestic violence. The Family Health Strategy (FHS) is fundamental in adolescent health care, although it has been little active in confronting violence against adolescents. Objective: This study aimed to describe the socioeconomic profile, identify maternal-fetal characteristics and situations of social vulnerability of young women with a history of teenage pregnancy and possible associations with the occurrence of domestic violence. Methodology: This is a cross-sectional study, with an interview of 100 adolescents between 13 and 19 years old with a history of pregnancy in 2018 in a poor neighborhood in Fortaleza - CE. A questionnaire with 57 questions was applied and the variables analyzed were related to socioeconomic profile, prenatal follow-up, childbirth and exposure to domestic violence during pregnancy. Chi-square test $\left(\chi^{2}\right)$ and Odds Ratio $(\mathrm{OR})$ were done in the analysis. Results: The average age of the interviewees was 17.5 years $(s d=1.65)$. The average family income was 1.18 minimum wage $(s d=0.83), 91 \%$ were black or brown, $57 \%$ were in a consensual union and $18 \%$ had a job. Regarding education, $71 \%$ stopped their studies, $46.5 \%$ of them did it before their first pregnancy and $35.2 \%$ stopped studying after becoming pregnant. In the prenatal follow-up, $96.3 \%$ had at least one medical appointment, $62.1 \%$ started in the first trimester and $69.1 \%$ had 6 or more medical appointments. Among the children, $7.6 \%$ and $6.4 \%$ were born premature and with underweight, respectively. The percentage of domestic violence was $26 \%$ and the main aggressor was the partner. Suffering domestic violence had a statistically significant association with low education (OR 4.06; 95\% Cl 1.27-12.97), lower maternal age (OR 4.2; 95\% Cl 1.43-12.32) and "history of newborn hospitalization" (OR 3.83; 95\% Cl 1.34-10.95). Conclusion: Adolescent mothers were in a context of social vulnerability and a part of them was a victim of violence during pregnancy, a situation associated with low schooling and a younger age of the adolescent. The negative consequences for the newborn's health tend to be more frequent in situations of violence. It was noted that adolescents had good prenatal attendance and that could have a positive influence on obstetric and neonatal outcomes. Pregnancy is often the young woman's first contact with the health service and access to prenatal care in the FHS for adolescents can reduce maternal and child complications and improve identification of domestic violence.
\end{abstract}

Keywords: Pregnancy in Adolescence; Primary Health Care; Gender and Health; Domestic Violence; Social Vulnerability.

\title{
Resumen
}

Introducción: El embarazo adolescente todavia tiene una alta incidencia en Brasil, incluso después de una gran reducción en las últimas décadas, y puede estar relacionado con situaciones de vulnerabilidad social y violencia doméstica. La Estrategia de Salud de la Familia (ESF) es fundamental en la atención de la salud de los adolescentes, sin embargo, se ha demostrado que es poco activa en el abordaje de la violencia en este grupo de edad. Objetivo: Este estudio tuvo como objetivo describir el perfil socioeconómico, identificar características materno-fetales y situaciones de vulnerabilidad social de mujeres jóvenes con antecedentes de embarazo adolescente y posibles asociaciones con la ocurrencia de violencia intrafamiliar. Metodología: Se trata de un estudio transversal, con entrevista a 100 adolescentes entre 13y 19 años con antecedentes de embarazo en 2018 en un barrio pobre de Fortaleza - CE. Se aplicó un cuestionario con 57 preguntas y las variables analizadas se relacionaron con perfil socioeconómico, seguimiento prenatal, parto y exposición a violencia doméstica durante el embarazo. En el análisis se realizaron pruebas de chi-cuadrado (X2) y Odds Ratio (OR). Resultados: La edad promedio de los entrevistados fue de 17,5 años $(d e=1,65)$. El salario familiar promedio fue de 1,18 salario mínimo (de $=0,83)$, el $91 \%$ eran negros o morenos, el $57 \%$ estaban en unión consensuada y el $18 \%$ tenían un trabajo. En cuanto a educación, el $71 \%$ había interrumpido sus estudios, el $46,5 \%$ de ellas lo hizo antes de su primer embarazo y el $35,2 \%$ dejó de estudiar tras quedar embarazada. En el seguimiento prenatal, el 96,3\% tuvo al menos una consulta, el 62,1\% inició en el primer trimestre y el $69,1 \%$ tuvo 6 o más consultas. Entre los niños, el 7,6\% y el 6,4\% nacieron prematuros y con bajo peso, respectivamente. El porcentaje de violencia doméstica fue del $26 \%$ y el principal agresor fue la pareja. El sufrimiento de violencia doméstica tuvo una asociación estadísticamente significativa con baja educación (OR 4.06; IC del 95\%: 1.27-12.97), menor edad materna (OR 4.2; IC del 95\% 1.43-12.32) y "antecedentes de hospitalización del recién nacido" (OR 3.83; 95\% Cl 1,34-10,95). Conclusión: Las madres adolescentes se encontraban en un contexto de vulnerabilidad social y una parte considerable fue víctima de violencia durante el embarazo, situación asociada a baja escolaridad y menor edad de la adolescente. Las consecuencias negativas para la salud del recién nacido fueron más frecuentes en situaciones de violencia. Se observó que las adolescentes tuvieron una buena asistencia prenatal y esto pudo haber tenido una influencia positiva en los resultados obstétricos y neonatales. El embarazo es a menudo el primer contacto de esta joven con el servicio de salud y el acceso a la atención prenatal en la ESF puede reducir las complicaciones materno infantiles y ayudar a identificar la violencia doméstica.

Palabras clave: Embarazo en Adolescencia; Atención Primaria de Salud; Género y Salud;Violencia Doméstica; Vulnerabilidad Social.

\section{INTRODUÇÃO}

A adolescência é um período de avanço na maturidade sexual e na formação da identidade, sendo uma fase de transição entre a infância e a fase adulta. Nesta fase, acontecem muitas transformações sociais, emocionais, biológicas, sendo a puberdade seu marco biológico de início¹. A adolescência compreende a faixa etária de 10 a 19 anos nas definições relacionadas à saúde ${ }^{1}$. A gravidez na adolescência tem ainda alta incidência no Brasil, quando comparada a países desenvolvidos, apesar da tendência à queda nos últimos anos ${ }^{2}$. A taxa de fecundidade em 2018 entre 15 e 19 anos por mil mulheres no mundo foi de 44 filhos, nas regiões mais desenvolvidas foi de 14 filhos, enquanto no Brasil foi de 62 filhos, com participação alta na fecundidade total ${ }^{2}$. Em Fortaleza (CE), houve queda na proporção de mães adolescentes de 17,3\% (2010) para 12,6\% (2019), entretanto tem havido um pequeno aumento na faixa etária entre 10 e 14 anos 3 . 
A gravidez na adolescência não é fenômeno recente, mas vem ocupando nas últimas décadas um lugar de relevância na saúde pública ${ }^{4}$. A inserção da mulher no mercado de trabalho, seu maior nível de escolaridade e a modificação da relação sexualidade/reprodução levaram a mudanças na maneira de compreender a gestação na adolescência, que passa a ser vista como problema social ${ }^{4}$. Entretanto, essas modificações não se expressam da mesma forma entre classes sociais e gêneros distintos, sendo a incidência da gestação na adolescência mais alta entre mulheres pobres e negras ${ }^{2,5}$.

A relação atribuída à idade materna como causa de desfechos negativos para o binômio mãebebê tem sido questionada, considerando que estes resultados estariam mais ligados às condições de vulnerabilidade social ${ }^{4}$. Vulnerabilidade social não é o mesmo que pobreza, embora a inclua. O conceito de vulnerabilidade social, segundo a UNESCO (Organização das Nações Unidas para a Educação, a Ciência e Cultura), é o resultado negativo da relação entre a disponibilidade de recursos materiais ou simbólicos e o acesso à estrutura de oportunidades sociais, econômicas e culturais providos pela sociedade. Esse resultado se traduz em debilidades para a mobilidade social dos indivíduos ${ }^{6}$.

Em contexto de vulnerabilidade social, a violência doméstica é condição presente entre adolescentes grávidas. Os adolescentes são um dos grupos da sociedade mais vulneráveis a sofrer violências ${ }^{7}$. A própria gestação pode ser uma porta de entrada para a ocorrência de violência doméstica, que pode trazer consequências negativas para a saúde da mãe e do feto, sendo um grave problema de saúde pública, que necessita ser prevenido ou identificado precocemente ${ }^{8}$. A violência no namoro entre jovens é frequente, apesar de ainda ser pouco investigada, tendo consequências e vivências diferentes para homens e mulheres, em que há perpetuação das desigualdades de gênero ${ }^{9}$. Na família, a violência doméstica ainda é considerada como parte da norma educativa, em um contexto marcado pela assimetria geracional ${ }^{10}$. Uma revisão integrativa sobre mães adolescentes vítimas de violência evidenciou que a Atenção Primária à Saúde (APS) através da Estratégia de Saúde da Família (ESF) auxilia na identificação do fenômeno da violência e a consulta de pré-natal mostrou-se um espaço facilitador do enfretamento à violência, já que a adolescente passa frequentar mais os serviços de saúde ${ }^{8}$. Entretanto, a ESF tem se mostrado pouco atuante no enfrentamento e prevenção das violências contra os adolescentes ${ }^{7}$.

Dessa forma, é importante ampliar os estudos sobre as distintas condições de vida das mães adolescentes e sobre a prevalência da violência doméstica na gestação e suas consequências no contexto da atenção primária.

Os objetivos deste estudo são descrever o perfil socioeconômico das jovens com histórico de gravidez na adolescência acompanhadas por unidade de saúde de bairro violento e pobre de Fortaleza, identificar as situações de vulnerabilidade social e as características materno-fetais e analisar a relação com a ocorrência de violência doméstica durante a gestação.

\section{MATERIAL E MÉTODOS}

Trata-se de um estudo de corte transversal, realizado com adolescentes moradoras do território adscrito à Unidade de Atenção Primária à Saúde Lineu Jucá do bairro Barra do Ceará em Fortaleza (CE). A cidade, capital do estado, está localizada no nordeste brasileiro, com aproximadamente 2.600 .000 habitantes e tem alto IDH (Índice de Desenvolvimento Humano) 0,732, embora ocupe o quarto pior índice entre as principais metrópoles brasileiras. O bairro da pesquisa é um dos mais violentos da cidade e tem IDH muito baixo $(0,215)$, um dos menores da capital11.

Aunidade de saúde em que foi realizada a pesquisa era composta por sete equipes de saúde da família completas (Médico, Enfermeiro, Técnico de Enfermagem, Dentista, Agente Comunitário de Saúde, Agente de Saúde Bucal), com a presença de residência de medicina de família e comunidade em cinco delas. 
A população alvo do estudo foi mulheres adolescentes entre 10 e 19 anos, gestantes no momento da entrevista ou que já tinham engravidado no período de 2015 até março de 2018. Foram identificadas no território 143 adolescentes com o perfil da pesquisa, a partir do prontuário eletrônico FastMedic da prefeitura de Fortaleza e por busca ativa no território realizada por Agentes Comunitários de Saúde e pela pesquisadora. Destas, primeiramente foram entrevistadas 10 adolescentes em estudo piloto a fim de aperfeiçoar e corrigir imperfeições do questionário. Não foi possível realizar a entrevista com 33 delas, pelos seguintes motivos: recusa em participar da entrevista (3); não encontrada no endereço (10); falecimento (1); mudança de endereço (17); violência no território (2). Dessa forma, a população entrevistada foi de 100 adolescentes moradoras do território analisado. A coleta de dados foi realizada durante o segundo semestre de 2018 e as entrevistas foram feitas pela própria pesquisadora nas instalações da unidade de saúde ou na casa das participantes, com duração média de 30 minutos, sendo garantida a privacidade.

Utilizou-se um questionário elaborado pela própria pesquisadora, a partir de revisão bibliográfica sobre o tema, com 57 perguntas fechadas e abertas, algumas com subtópicos. Para o presente artigo, foram coletadas informações do perfil socioeconômico das participantes (idade, raça, religião, estado civil, escolaridade, ocupação, renda), estrutura familiar, violência na gestação, experiência sexual e reprodutiva, informações sobre período da gestação e sobre o recém-nascido, planejamento da gestação e dados sobre o pai da criança. No mesmo questionário foram obtidos dados sobre condições de nascimento (peso, idade de nascimento, problemas de saúde, internação, amamentação) de 77 filhos das adolescentes, sendo considerada na análise a criança da última gestação.

Para caracterização da população utilizou-se estatística descritiva e para a análise da variável resposta violência doméstica foi utilizado teste qui-quadrado $\left(x^{2}\right)$ e Odds Ratio (OR), admitindo-se nível de significância 5\%. A tabulação dos dados foi realizada no Microsoft Office Excel 365 . $O$ tratamento estatístico das informações foi feito no software livre R versão 3.6.1 $1^{12}$.

O estudo foi conduzido segundo os preceitos éticos. As adolescentes e seus responsáveis foram informados sobre a pesquisa e tiveram as dúvidas esclarecidas. Foram entregues dois tipos de termo de consentimento livre e esclarecido: um para as adolescentes com 18 e 19 anos, e outro para os responsáveis das adolescentes menores de idade. Um termo de assentimento foi preenchido pelas adolescentes com menos de 18 anos. O projeto foi aprovado pelo Comitê de Ética em Pesquisa do Hospital Universitário Walter Cantídio da Universidade Federal do Ceará / HUWC - UFC. A pesquisa seguiu as orientações da Resolução 466/12 do Conselho Nacional de Saúde que trata da pesquisa com seres humanos.

\section{RESULTADOS}

Responderam ao questionário adolescentes entre 13 e 19 anos, pois não havia participantes de 10 a 12 anos com histórico de gravidez no período das entrevistas. Aidade média das adolescentes entrevistadas foi 17,53 anos com desvio padrão (dp) de 1,65 e a idade média no momento da gestação avaliada foi 17,2 anos ( $d p=1,71)$. Quase metade (42\%) estavam na adolescência tardia (18 e 19 anos) e 10\% tinham 13 ou 14 anos no momento da entrevista.

A maioria das participantes tinham religião (64\%), sendo mais citada a católica. A renda familiar média foi 1,18 ( $\mathrm{dp}=0,83)$ salário-mínimo (salário-mínimo era 954 reais no ano da pesquisa), com valor máximo de 4 salários-mínimos e $17 \%$ não sabiam informar a renda familiar. A principal ocupação relatada foi costureira 
(50\%), a maioria em empregos informais. O tempo médio de união consensual foi de 34 meses. Em $46 \%$ dos domicílios moravam três pessoas, em $13 \%$ viviam quatro e em $35 \%$ habitavam cinco ou mais. A maioria das adolescentes (65\%) moravam com o companheiro, que também era o pai da criança. Mais da metade (55\%) moravam somente com companheiro e filho(a), sem dependência financeira dos familiares. O histórico de gestação na adolescência estava presente em $86 \%$ das famílias, sendo a mãe (52\%) a mais citada (Tabela 1).

Quanto à escolaridade, maior parcela das adolescentes estava fora da escola (71\%) sendo o principal motivo alegado por elas "a falta de interesse". Ressalta-se que 13 jovens (18,3\%) já haviam concluído o ensino médio, mas não ingressaram no ensino técnico ou superior. Dentre aquelas que pararam de estudar e não concluíram o ensino médio, 33 adolescentes $(46,5 \%)$ interromperam os estudos antes da gestação e 25 adolescentes (35,2\%) pararam de estudar depois de engravidar. Entre aquelas inseridas na escola (29\%), foi encontrada uma importante distorção idade-série (2 anos ou mais de atraso escolar): 17 adolescentes apresentaram distorção (58,6\%).

Tabela 1. Características sociodemográficas das adolescentes. Fortaleza, 2018.

\begin{tabular}{|c|c|c|c|}
\hline Variáveis & $\mathbf{N}$ & $\%$ & IC 95\% \\
\hline \multicolumn{4}{|l|}{ Idade (anos) } \\
\hline $13-17$ & 58 & 58 & $48,2-67,2$ \\
\hline $18-19$ & 42 & 42 & $32,8-51,8$ \\
\hline \multicolumn{4}{|l|}{ Raça } \\
\hline Branca & 6 & 6,3 & $2,86-12,84$ \\
\hline Negra & 9 & 9,2 & $4,96-16,7$ \\
\hline Parda & 82 & 84,5 & $76,04-90,4$ \\
\hline \multicolumn{4}{|l|}{ Religião } \\
\hline Católica & 33 & 34 & $25,36-43,89$ \\
\hline Evangélica & 28 & 28,9 & $20,79-38,55$ \\
\hline Sem religião & 36 & 37,1 & $28,16-47,04$ \\
\hline \multicolumn{4}{|l|}{ Estado Civil } \\
\hline Solteira & 35 & 35 & $26,36-44,74$ \\
\hline Casada & 8 & 8 & $41,09-14,99$ \\
\hline União Consensual & 57 & 57 & $47,21-66,26$ \\
\hline \multicolumn{4}{|l|}{ Renda familiar } \\
\hline$<1 \mathrm{SM}^{\star}$ & 17 & 20,5 & $13,2-30,37$ \\
\hline $1 \mathrm{SM}$ & 45 & 54,2 & $43,55-64,51$ \\
\hline$>1 \mathrm{SM}$ & 21 & 25,3 & $17,18-35,6$ \\
\hline \multicolumn{4}{|l|}{ Trabalha } \\
\hline Sim & 18 & 18 & $11,7-26,67$ \\
\hline Não & 82 & 82 & $73,33-88,3$ \\
\hline \multicolumn{4}{|c|}{ Gravidez na adolescência em familiares } \\
\hline Mãe & 52 & 60,4 & $42,31-61,53$ \\
\hline Irmã & 32 & 37,2 & $23,67-41,66$ \\
\hline Avó & 11 & 12,8 & $6,25-18,63$ \\
\hline Prima & 32 & 37,2 & $23,67-41,66$ \\
\hline Tia & 12 & 14 & $6,99-19,81$ \\
\hline \multicolumn{4}{|l|}{ Escolaridade } \\
\hline Ensino Fundamental & 64 & 64 & 54,23 \\
\hline Ensino Médio & 35 & 35 & $26,36-44,74$ \\
\hline Ensino Superior & 1 & 1 & $0,17-5,45$ \\
\hline
\end{tabular}

*SM (salário-mínimo) 
A idade média da primeira menstruação (menarca) foi 12 anos $(\mathrm{dp}=1,44)$ e a idade média da primeira relação sexual (sexarca) foi 14,21 anos ( $d p=1,5)$. Quando questionadas sobre abordagem da sexualidade, $48 \%$ das jovens tiveram tema abordado por familiares e para $57 \%$ foi discutido na escola. Evidenciouse que a maioria das adolescentes, $69 \%$, não fazia uso de método contraceptivo antes de engravidar. Entre aquelas que faziam uso $(31 \%)$, os métodos mais citados foram: preservativo masculino $(38,8 \%)$, anticoncepcional oral $(32,2 \%)$ e anticoncepcional injetável mensal $(29 \%)$.

Não houve relato de parto por fórceps. Com relação a abortamento, 10\% (10 casos) das jovens relataram ter sofrido abortos, sendo seis deles informados como provocados. Dentre as jovens com histórico de gravidez e que não estavam grávidas (81\%) no momento da entrevista, 96,3\% realizaram pelo menos uma consulta de pré-natal e $69,1 \%$ fizeram 6 ou mais consultas, que é o mínimo recomendado pelo Ministério da Saúde (Tabela 2). Complicações na gestação foram relatadas por $48 \%$ das entrevistadas, sendo as principais: infecção do trato urinário (26\%), anemia (11\%), doença hipertensiva específica da gravidez $(9 \%)$, sangramento transvaginal $(5 \%)$ e sífilis $(3 \%)$.

Tabela 2. Dados sobre a gestação das adolescentes.Fortaleza, 2018.

\begin{tabular}{|c|c|c|c|}
\hline Variáveis & $\mathbf{N}$ & $\%$ & IC 95\% \\
\hline \multicolumn{4}{|c|}{ Gestante no momento da entrevista } \\
\hline Sim & 19 & 19 & $12,51-27,77$ \\
\hline Não & 81 & 81 & $72,22-87,48$ \\
\hline \multicolumn{4}{|l|}{ Planejada } \\
\hline Sim & 22 & 22 & $15,00-31,07$ \\
\hline Não & 78 & 78 & $68,93-84,99$ \\
\hline \multicolumn{4}{|l|}{ Desejada } \\
\hline Sim & 67 & 67 & $57,30-75,43$ \\
\hline Não & 33 & 33 & $24,56-42,69$ \\
\hline \multicolumn{4}{|l|}{ Tipo de Parto } \\
\hline Vaginal & 65 & 65 & $55,25-73,63$ \\
\hline Cesáreo & 35 & 35 & $26,36-44,74$ \\
\hline \multicolumn{4}{|c|}{ Início do Pré-Natal } \\
\hline $1^{\circ}$ trimestre & 59 & 62,1 & $51,53-71,69$ \\
\hline $2^{\circ}$ trimestre & 33 & 34,7 & $25,92-44,73$ \\
\hline $3^{\circ}$ trimestre & 3 & 3,2 & $1,08-8,87$ \\
\hline \multicolumn{4}{|c|}{ Consulta de pré-natal } \\
\hline $0-2$ consultas & 6 & 7,4 & $3,48-15,41$ \\
\hline 3-5 consultas & 18 & 22,2 & $14,73-32,78$ \\
\hline 6 ou mais & 56 & 69,1 & $59,23-78,93$ \\
\hline \multicolumn{4}{|c|}{ Grau de Gestação } \\
\hline 1 gestação & 73 & 73 & $63,56-80,73$ \\
\hline 2 gestações & 23 & 23 & $15,84-32,15$ \\
\hline 3 gestações & 1 & 1 & $0,17-5,44$ \\
\hline 4 gestações & 3 & 3 & $1,02-8,45$ \\
\hline \multicolumn{4}{|c|}{ Amamentação por 6 meses ou mais } \\
\hline Sim & 38 & 49,4 & $38,48-60,28$ \\
\hline Não & 39 & 50,6 & $39,72-61,51$ \\
\hline Exclusivo & 20 & 26 & $17,49-36,74$ \\
\hline
\end{tabular}


Com relação aos recém-nascidos, foi evidenciado que 32,5\% apresentaram algum tipo de problema de saúde no nascimento e $28,7 \%$ teve que permanecer internado. O principal problema de saúde citado foi icterícia fisiológica (15,6\%), seguido de desconforto respiratório (6,5\%). As crianças prematuras representaram $7,6 \%$ dos casos. Quanto ao peso ao nascer, cinco crianças $(6,4 \%)$ nasceram com baixo peso (menos de 2500 gramas). Houve apenas um óbito fetal relatado.

Quase todas as adolescentes iniciaram amamentação $(97,4 \%)$. A mediana para aleitamento materno foi 135 dias, já a mediana para aleitamento materno exclusivo (AME) foi 90 dias (Tabela 2).

Os dados relacionados ao pai das crianças foram obtidos através do relato das adolescentes (Tabela 3). A idade média do pai foi 22,5 anos ( $d p=4,33$ ), com idade máxima de 42 anos.

A violência doméstica sofrida na gestação foi relatada por $26 \%$ (26 casos) das adolescentes, sendo a violência psicológica a mais prevalente com 23 relatos $(88 \%)$, seguida da violência física $(34,6 \%)$ e sexual (13\%). O companheiro foi o principal agressor em 14 casos (56\%), seguido de pai e mãe que foram agressores em $20 \%$ dos casos cada um (Tabela 4). Com relação à violência e escolaridade, dentre os casos de violência relatados, 17 adolescentes já haviam interrompido os estudos e entre aquelas que ainda estudavam, todas (seis) apresentavam distorção idade-série.

Tabela 3. Características do pai da criança. Fortaleza, 2018.

\begin{tabular}{|c|c|c|c|}
\hline Variáveis & $\mathbf{N}$ & $\%$ & IC $95 \%$ \\
\hline \multicolumn{4}{|c|}{ Mora com mãe da criança } \\
\hline Sim & 65 & 65 & $55,25-73,63$ \\
\hline Não & 35 & 35 & $26,36-44,74$ \\
\hline \multicolumn{4}{|l|}{ Estuda } \\
\hline Sim & 12 & 12,4 & $72,20-20,38$ \\
\hline Não & 85 & 87,6 & $79,61-92,78$ \\
\hline \multicolumn{4}{|l|}{ Trabalha } \\
\hline Sim & 71 & 73,2 & $63,62-81,00$ \\
\hline Não & 26 & 26,8 & $18,99-36,37$ \\
\hline \multicolumn{4}{|l|}{ Idade do pai } \\
\hline$<20$ anos & 22 & 22,5 & $15,32-31,65$ \\
\hline 20-29 anos & 68 & 69,3 & $59,67-77,63$ \\
\hline 30 ou mais & 8 & 8,2 & $4,19-15,28$ \\
\hline \multicolumn{4}{|l|}{ Escolaridade } \\
\hline Ensino Fundamental & 32 & 40 & $29,96-50,95$ \\
\hline Ensino Médio & 44 & 55 & $44,12-65,42$ \\
\hline Ensino Superior & 2 & 2,5 & $0,68-8,66$ \\
\hline Sem alfabetização & 2 & 2,5 & $0,68-8,66$ \\
\hline \multicolumn{4}{|c|}{ Assumiu paternidade } \\
\hline Sim & 66 & 84,6 & $75,00-90,97$ \\
\hline Não & 12 & 15,4 & $9,02-24,99$ \\
\hline \multicolumn{4}{|c|}{ Ajuda financeiramente } \\
\hline Sim & 59 & 74,7 & $64,10-82,97$ \\
\hline Não & 20 & 25,3 & $17,02-35,89$ \\
\hline
\end{tabular}


Tabela 4. Variáveis relacionadas com violência doméstica na gestação. Fortaleza, 2018.

\begin{tabular}{|c|c|c|c|c|}
\hline \multirow{2}{*}{ Variáveis } & \multicolumn{2}{|c|}{ Violência Doméstica (n) } & \multirow{2}{*}{ p-valor } & \multirow{2}{*}{ OR (IC 95\%) } \\
\hline & Sim & Não & & \\
\hline Escolaridade da adolescente & & & 0,025 & $4,06(1,27-12,97)$ \\
\hline Ensino Fundamental & 22 & 42 & & \\
\hline Ensino Médio & 4 & 31 & & \\
\hline Idade da adolescente & & & 0,006 & $4,2(1,43-12,32)$ \\
\hline 13-17 anos & 21 & 37 & & \\
\hline 18-19 anos & 5 & 37 & & \\
\hline Renda familiar & & & 0,54 & $1,73(0,51-5,88)$ \\
\hline$<=1 \mathrm{SM}^{*}$ & 18 & 44 & & \\
\hline$>1 \mathrm{SM}$ & 4 & 17 & & \\
\hline Complicação na gestação & & & 0,13 & $2,03(0,8-5,2)$ \\
\hline Sim & 16 & 34 & & \\
\hline Não & 9 & 39 & & \\
\hline $\mathbf{R N}^{\star \star}$ nasceu com algum problema de saúde & & & 0,07 & $2,86(1,03-7,96)$ \\
\hline Sim & 11 & 15 & & \\
\hline Não & 11 & 43 & & \\
\hline História de internação do $\mathbf{R N}^{\star \star}$ & & & 0,02 & $3,83(1,34-10,95)$ \\
\hline $\operatorname{Sim}$ & 11 & 12 & & \\
\hline Não & 11 & 46 & & \\
\hline
\end{tabular}

\section{DISCUSSÃO}

O perfil socioeconômico das jovens deste estudo evidencia uma condição de grande vulnerabilidade social, com famílias de baixa renda, em sua maioria de raça negra ou parda e com baixa escolaridade. No Brasil, 12,6\% dos jovens de 15 a 19 anos vivem em união consensual ou casamento e no Ceará esse índice representa $18,3 \%{ }^{13}$. Estes índices são bastante inferiores à porcentagem encontrada nesta pesquisa (65\%), possivelmente devido ao estudo ter abordado somente adolescentes com histórico de gestação. Esta porcentagem de união estável ou casamento encontrada mostra uma saída precoce das jovens da casa dos pais. Como evidenciado nos resultados, as jovens deste estudo provêm de famílias onde a gravidez na adolescência é parte da realidade delas, situação relatada como frequente na literatura ${ }^{14,15}$.

Quanto à escolaridade, é fundamental refletir criticamente sobre a responsabilização que tem sido atribuída à gravidez na adolescência pelo abandono escolar. Neste estudo, a maioria das adolescentes já haviam deixado a escola antes mesmo de engravidar. Assim, a relação escolaridade e gravidez na adolescência não é uma equação simples, com diferenças entre os gêneros e devem ser avaliadas as situações de vulnerabilidade social que levam a adolescente a sair da escola. O percurso escolar das classes populares é muitas vezes breve e marcado por várias repetências e interrupções e algumas razões apontadas para a evasão escolar são: mudanças de domicílio, precariedade das redes de ensino público, a violência nas áreas das escolas, inserção no mercado de trabalho (mais frequente entre os homens), e a internalização feminina para cuidar da casa e dos irmãos ${ }^{4,16}$. Essas jovens com baixa escolaridade ingressam no mercado de trabalho, mas de forma informal e em posições bastante desqualificadas, não conseguindo vislumbrar uma ascensão social como possibilidade de futuro ${ }^{16}$. 
A idade média da sexarca do nosso estudo coincidiu com a idade encontrada em pesquisa nacional sobre sexualidade na juventude de 14,8 anos ${ }^{9}$. As adolescentes entrevistadas demoraram em média 2,8 anos para ter a primeira relação sexual após a menarca e demoraram em média 2,6 anos entre a primeira relação sexual e a primeira gestação. Assim, o sistema de saúde, a família e as instituições escolares tiveram mais de 2 anos para realizar orientações sobre educação sexual antes da gestação, mesmo após o início da vida sexual. A abordagem da sexualidade juvenil em escolas, serviços de saúde e famílias ainda é um desafio ${ }^{4,16}$. A temática sexualidade adolescente é ainda abordada pelas políticas públicas sob uma perspectiva negativa e "controlista", sendo associada no geral à violência, às doenças sexualmente transmissíveis e à gravidez indesejada. Dessa forma, é fundamental organizar uma agenda positiva, com a construção da perspectiva dos adolescentes como sujeitos de direitos, inclusive em relação aos direitos sexuais ${ }^{17}$.

No presente estudo, quase um terço das gestações das adolescentes foram planejadas e dois terços desejadas. Isso mostra que, ao contrário do que é propagado, a gravidez na adolescência não é necessariamente indesejada ou não planejada e pode estar associada a um cenário de busca por valorização da mulher e por reconhecimento social ${ }^{14}$. Em contexto social de poucas oportunidades, a gravidez para muitas mulheres passa a ser uma fonte de reconhecimento social e o discurso moral, que coloca a jovem como vítima imatura e irresponsável, resulta em políticas pouco direcionadas para os indivíduos e sua situação de vida ${ }^{5}$. Assim é fundamental rever as políticas públicas sobre a temática e considerar as diferenças nas histórias de vida dessas adolescentes e ampliação dos futuros possíveis ${ }^{14}$.

Outras investigações com delineamentos qualitativos poderiam ajudar a elucidar melhor essa realidade apontada pelo presente estudo.

A taxa de utilização de métodos contraceptivos pelas adolescentes foi baixa em nosso estudo, semelhante ao observado em outras capitais, assim como o conhecimento dos métodos mostrou não garantir seu uso ${ }^{5,14,15}$. Outro estudo nacional, que corrobora com nossos resultados, encontrou que mais de $80 \%$ das adolescentes não usavam contracepção ou usavam irregularmente quando engravidaram, no qual a maioria das participantes relatou conhecimento sobre preservativos (91\%), pílula anticoncepcional oral combinada $(83 \%)$ e injetáveis contraceptivos $(76 \%)^{18}$. Índice de aborto deste estudo foi semelhante ao encontrado na literatura $(9,2 \%)^{9}$.

Esta pesquisa constatou que houve acesso às consultas de pré-natal pela maior parte das adolescentes, com frequência nas consultas de pré-natal semelhante à porcentagem nacional para adolescentes, a maioria com 6 ou mais consultas $(69,1 \%)$ e com início no primeiro trimestre $(62,1 \%)$, apesar desse número ainda ser considerado baixo. No Brasil, a cobertura do pré-natal detectada foi elevada $(98,7 \%)$, com menor proporção de início precoce entre adolescentes, 10 a 14 anos (51,7\%) e entre 15 e 19 anos (66,7\%) e os índices de comparecimento a 6 ou mais consultas foram 62,4\% para aquelas entre 15 e 19 anos $^{19}$.

$O$ peso da maioria dos recém-nascidos foi adequado para idade gestacional e o índice de prematuridade foi pequeno na população estudada, abaixo do índice nacional de parto prematuro de 11,3\% para todas as faixas etárias ${ }^{9}$. Neste estudo, apesar de quase metade (48\%) ter relatado complicações na gestação, no geral foram complicações comuns encontradas em gestações de todas as faixas etárias ${ }^{20}$, pois $77 \%$ delas foram infecção urinária e anemia. Com relação aos problemas de saúde no nascimento da criança, a icterícia foi o achado mais frequente, sendo que a icterícia neonatal é uma condição comum e na maioria dos casos reflete uma adaptação fisiológica do recém-nascido ao metabolismo da bilirrubina. 
É importante notar que neste estudo a maior parte das gestantes adolescentes relacionou-se adequadamente com o sistema de saúde, visto que a maioria iniciou o pré-natal no primeiro trimestre e realizou mais de 6 consultas. Isso pode ter influenciado positivamente os desfechos obstétricos e neonatais.

Alguns estudos sobre gestação na adolescência têm como enfoque possíveis consequências negativas devido a idade da mãe para o binômio mãe-bebê, como maior risco de aborto, prematuridade, baixo peso ao nascer, aumento da mortalidade materna e infanti ${ }^{21,22}$. Entretanto, outros estudos têm questionado a relação entre os desfechos negativos e a idade materna, considerando que estes desfechos estariam mais ligados às condições de vulnerabilidade social (situação socioeconômica, acesso aos serviços de saúde) das adolescentes do que à idade propriamente ${ }^{4,23}$. Os riscos de resultados negativos seriam os mesmos para mulheres de 15 a 19 anos quando comparadas com mulheres mais velhas e seriam realmente maiores para gestantes com idade entre 10 e 14 anos $^{4,19}$.

A porcentagem de aleitamento materno exclusivo (AME), entre os menores de seis meses de idade neste estudo (26\%) foi menor do que a porcentagem nacional de $38,6 \%{ }^{24}$, o que pode significar uma dificuldade em manter o aleitamento exclusivo entre adolescentes e uma deficiência na orientação sobre o tema durante o pré-natal.

A maioria dos pais das crianças relatou ter emprego, em contraste com o encontrado para as mães adolescentes, que em maior número nem estudavam e nem trabalhavam. Isso pode demonstrar a presença ainda marcante nas classes populares da responsabilização das mulheres pelos cuidados com casa e filhos(as), com consequente internalização, e dos homens pelo sustento da família, que desde cedo devem procurar emprego, o que pode levar ao abandono precoce da escola por estes últimos ${ }^{4,9}$. Em torno de um quinto dos pais também eram adolescentes, sendo o tema da paternidade juvenil ainda pouco abordado na literatura ${ }^{25,26}$.

As mulheres tiveram relacionamentos afetivos com homens em média cinco anos mais velhos, inclusive maior que a média encontrada em estudo nacional ${ }^{9}$, o que pode demonstrar uma tendência das jovens a se relacionar com homens mais velhos, havendo, em alguns casos, a necessidade da abordagem de aspectos jurídicos, principalmente com relação às jovens com idade entre 10 e 14 anos. As adolescentes que têm parceiros mais velhos do sexo masculino são mais propensas a iniciar atividade sexual e engravidar mais cedo e ser vítima de violência doméstica do que aquelas com parceiros de idade semelhante ${ }^{25}$.

Quase um terço das adolescentes deste estudo foram vítimas de violência doméstica durante a gestação, sendo mais citada a violência psicológica, seguida de violência física e sexual, corroborando com achados da literatura ${ }^{8,9}$. A violência doméstica é condição frequente entre adolescentes grávidas, com aumento da morbidade pós-parto materna e neonatal, sendo uma particular preocupação para o Sistema Único de Saúde (SUS). A desigualdade de gênero presente em nossa sociedade, contribui na perpetuação dessa violência ${ }^{27}$. Os pais também foram relatados como agressores das adolescentes, sendo a família permeada por relações de poder e assimetria geracional, em que a violência é ainda utilizada como medida educativa e disciplinar ${ }^{10}$.

Verificou-se ainda uma associação entre a ocorrência de violência doméstica com a escolaridade da adolescente e a idade materna. Abandono da escola ou atraso escolar foi condição frequente entre as adolescentes vítima de violência, evidenciando que as chances de atraso escolar entre as adolescentes que sofreram violência foram 4,06 vezes maiores em relação àquelas não expostas à violência. As adolescentes mais jovens, com idade entre 13 e 17 anos, apresentaram chances de ocorrência de violência 4,2 vezes 
aquelas expostas na idade de 18 e 19 anos, mostrando que o grupo mais jovem apresenta provavelmente uma maior vulnerabilidade.

A história de internação do recém-nascido também apresentou associação com ser vítima de violência doméstica. As chances de ocorrência de internação de recém-nascidos entre aqueles cuja mãe adolescente sofreu violência doméstica são 3,83 vezes maiores. A variável "recém-nascido nasceu com algum problema de saúde", apesar de não ter apresentado significância estatística (com p-valor limítrofe), se mostrou 2,86 vezes mais presente entre as vítimas de violência. As outras variáveis relacionadas às condições socioeconômicas e características materno-infantis não tiveram associação estatisticamente significativa com a violência doméstica.

A violência doméstica sofrida na adolescência é um problema social que no âmbito da ação da ESF só recentemente começa a ser objeto de estudo, demarcando um campo de atuação desafiador para as políticas públicas, consequentemente para a atenção primária ${ }^{8}$. $O$ enfrentamento da violência envolvendo adolescentes requer um rompimento do modelo de saúde fragmentado, com vistas à construção de um trabalho interprofissional e intersetorial, com a necessidade do incremento de debates e pesquisas sobre o tema, sintonizadas com a realidade da saúde pública brasileira ${ }^{7,8,27}$.

A ESF volta-se para grupos sociais mais vulneráveis, seguindo os princípios doutrinários do SUS, como a integralidade, e os atributos essenciais da APS, como a longitudinalidade, que na adolescência ganham grande importância, pois permitem uma promoção de saúde que respeite as especificidades desse período da vida ${ }^{28}$.

Este estudo tem limitações que são próprias dos estudos transversais e não pode assim avaliar causalidade. Outras limitações são: dados relacionados ao parceiro e ao filho foram obtidos indiretamente pelas respostas das adolescentes e a porcentagem de violência doméstica pode ser maior que a encontrada, pois algumas adolescentes que não conseguimos entrevistar tinham condição de alta vulnerabilidade social, podendo ser vítimas de violência doméstica.

\section{CONCLUSÃO}

As mães adolescentes estavam inseridas em um contexto de vulnerabilidade social e uma parte considerável foi vítima de violência durante a gestação. As adolescentes mais vulneráveis, com baixa escolaridade, tiveram maior chance de sofrer violência doméstica. Grande parte dos variados riscos relacionados com a gestação na adolescência estavam presentes antes mesmo das jovens engravidarem e as consequências negativas para saúde da adolescente e do recém-nascido tendem a ser mais frequentes em situações de violência. O acesso adequado ao sistema de saúde durante o pré-natal pode ter impacto na redução da morbidade materna e neonatal.

A redução da vulnerabilidade social, acesso a pré-natal de qualidade e garantia dos direitos sexuais dos adolescentes são ações primordiais para possibilitar aos jovens oportunidades e escolhas sobre o seu futuro, redução dos índices de complicações materno-fetais e prevenção e identificação precoce de casos de violência. A gestação é, muitas vezes, o primeiro contato dessa jovem com o serviço de saúde, sendo fundamental garantir acolhimento e atenção às especificidades da saúde do adolescente, superando visões estigmatizadas. Neste contexto, também é importante para redução da violência, a superação das assimetrias de gênero e geracionais, em que mulheres, crianças e adolescentes são as principais vítimas. 
Faz-se necessária a garantia na atenção primária à saúde de um atendimento interdisciplinar e intersetorial para a adolescente grávida e sua família. É importante uma ressignificação da imagem do adolescente e uma promoção à saúde junto a esse segmento, com a implementação de práticas alternativas e criativas que valorizem o protagonismo juvenil. Há ainda uma lacuna de estudos com relação ao cuidado das adolescentes grávidas em situação de violência na APS, sendo importante a realização de mais pesquisas inclusive com delineamento longitudinal.

\section{Agradecimentos}

Este artigo é parte do Trabalho de Conclusão da Residência Médica em Medicina de Família e Comunidade da autora principal. Agradecemos a grande ajuda recebida dos funcionários do CUCA Barra e dos profissionais de saúde da Unidade de Atenção Primária à Saúde Lineu Jucá, em especial das Agentes Comunitárias de Saúde (ACS) Maria das Graças da Silva (Márcia), Maria Vanderli Prudêncio da Cruz, Cícera Regina Silva de Lima Melo e Eridam de Sousa Ferreira, que muito auxiliaram no trabalho de campo, na coleta de dados e na localização das participantes no território estudado.

\section{Conflitos de Interesse:}

As autoras declaram não haver conflitos de interesse.

\section{REFERÊNCIAS}

1. World Health Organization. WHO recommendations on adolescent sexual anda reproductive health and rights. WHO. 2018 .

2. United Nations Population Fund. UNFPA. Situação da População Mundial 2019. Um trabalho inacabado: a busca por direitos e escolhas para todos e todas. 2019. Disponível em: <https://brazil.unfpa.org/pt-br/publications/situacao-da-populacao-mundial-2019>. Acesso em: agosto de 2019.

3. Bárbara Câmara. Mais de 4 mil crianças em Fortaleza nasceram de mães com idade entre 10 e 19 anos em 2019 [Internet]. Ceará:G1: [atualizado em 2020 Jan. 11; citado em 2020 Jul. 15]. Disponível em: <https://g1.globo.com/ce/ceara/noticia/2020/01/11/mais-de-4mil-criancas-em-fortaleza-nasceram-de-maes-comidade-entre-10-e-19-anos-em-2019.ghtml>.

4. Heilborn ML, Salem T, Rohden F, Brandão E, Knauth D, Víctora C, et al. Aproximações socioantropológicas sobre a gravidez na adolescência. Horiz Antropológicos. junho de 2002;8(17):13-45.

5. Aquino, Estela Maria L. de et al. Adolescência e reprodução no Brasil : a heterogeneidade dos perfis sociais. Cad Saúde Pública. 2003;19 (Supl 2):377-88.

6. Ambromoway, Miriam, et al. Juventude, violência e Vulnerabilidade Social na América Latina; desafios para políticas públicas. Brasília. UNESCO. BID. 2002. $192 \mathrm{p}$.

7. Vieira Netto MF, Deslandes SF. As Estratégias da Saúde da Família no enfrentamento das violências envolvendo adolescentes. Ciênc Saúde Coletiva. 2016;21(5):1583-96.

8. Macedo CM, Miura PO, Barrientos DMS, Lopes GA, Egry EY. Coping strategies for domestic violence against pregnant female adolescents: integrative review. Rev Bras Enferm. 2018;71(suppl 1):693-699.

9. Minayo MC de S, Assis SG de, Njaine K, organizadores. Amor e violência: um paradoxo das relações de namoro e do "ficar" entre jovens brasileiros. Rio de Janeiro, RJ: Editora Fiocruz; 2011.236 p.

10. Gomes NP, Diniz NMF, Araújo AJS, Coelho TMF. Compreendendo a violência doméstica a partir das categorias gênero e geração. Acta Paul Enferm. 2007;20(4):504-8.

11. Programa das Nações Unidas para o Desenvolvimento. PNUD. Instituto de Pesquisa Econômica e Aplicada. IPEA. Fundação João Pinheiro. Atlas de Desenvolvimento Humano do Brasil de 2013. 2013. Disponível em: <http://www.atlasbrasil.org.br/2013/>. Acesso em: julho de 2019. 
12. R Core Team (2019) R: A language and environment for statistical computing. R Foundation for Statistical Computing, Vienna, Austria. URL https://www.R-project.org/.

13. Instituto de Pesquisa e Estratégia Econômica do Ceará. Indicadores Sociais do Ceará. Fortaleza. IPECE. 2018. Disponível em:<https:// www.ipece.ce.gov.br/wp-content/uploads/sites/45/2019/01/Indicadores_Sociais_2017.pdf>. Acesso em: julho de 2019.

14. Vieira EM, Bousquat A, Barros CRDS, Alves MCGP. Adolescent pregnancy and transition to adulthood in young users of the SUS. Rev Saúde Pública 2017;51:1-11.

15. Marino, Jennifer L; Lewis, Lucy N; Bateson, Deborah; Hickey, Martha and Skinner, SRachel. Teenage mothers [online]. Australian Family Physician, Vol. 45, No. 10, Oct 2016:712-717.

16. Brandão ER. Gravidez na adolescência: um balanço bibliográfico. In: Heilborn ML, Aquino EML, Knauth D, Bozon M, organizadores. O aprendizado da sexualidade: um estudo sobre reprodução e trajetórias sociais de jovens brasileiros. Rio de Janeiro: Garamond; 2006.

17. Leite, V. Sexualidade Adolescente como direito? A visão de formuladores de políticas públicas. Rio de Janeiro. Editora Eduerj. 2013. Coleção Sexualidade, gênero e sociedade.

18. Brito, M. B., Alves, F. S. S., Souza, M. Q., \& Requião, S. R. (2018). Low Level of Knowledge of Contraceptive Methods among Pregnant Teens in Brazil. Journal of Pediatric and Adolescent Gynecology, 31(3), 281-284.

19. Viellas EF, Domingues RMSM, Dias MAB, Gama SGN da, Theme Filha MM, Costa JV da, et al. Assistência pré-natal no Brasil. Cad Saúde Pública. agosto de 2014;30(suppl 1):S85-100.

20. Varela PLR, Oliveira RR, Melo EC, Mathias TAF. Pregnancy complications in Brazilian puerperal women treated in the public and private health systems. Rev. Latino-Am. Enfermagem. 2017;25:e2949.

21. Silva PC, Barbosa TLSM, Farias RAR, Lopes MLH, Silva EL, Nunes FBBF. Influence of maternal age in perinatal conditions in live births of São Luís, Maranhão. Rev Fun Care Online. 2020 jan/dez; 12:292-299.

22. Karataşı ı Vanmaz AG, İnan AH, Budak A, Beyan E. Maternal and neonatal outcomes of adolescent pregnancy. J Gynecol Obstet Hum Reprod. 2019;48(5):347-350.

23. Farias RV, SoaresCFS e, AraújoR da S, AlmeidaVRS de, LeitãoD de S, SantosJS dos, Santos LS, NogueiraSD de A, Morais AC, Oliveira CBF. Gravidez na adolescência e o desfecho da prematuridade: uma revisão integrativa de literatura. REAS [Internet]. 13ago.2020 [citado 18out.2020];(56):e3977.

24. Global Breastfeeding Collective (2017). Nurturing the health and wealth of nations: The investment case for breastfeeding. UNICEF/ $\mathrm{WHO} / 1000$ days/Alive \& Thrive.

25. Wang, C S, Chou, P. Characteristics of males who father babies born to adolescents versus older adult women in Taiwan. Journal of Adolescent Health 2001; 28: 509-512.

26. Bamishigbin ON Jr, Dunkel Schetter C, Stanton AL. The antecedents and consequences of adolescent fatherhood: A systematic review. Soc Sci Med. 2019;232:106-119.

27. Camargo, N. F. et al. Adolescentes grávidas vítimas de violência: um desafio a ser enfrentado na Atenção Básica TT - Pregnant adolescents victims of violence: a challenge to be faced in primary care. BIS. Boletim do Instituto de Saúde. 2017;2(17):29-36.

28. Brasil. Ministério da Saúde. Portaria oㅜ 2.436, de 21 de setembro de 2017. Aprova a política nacional de atenção básica, estabelecendo a revisão de diretrizes para a organização da atenção básica, no âmbito do sistema único de saúde (SUS). Diário Oficial da União. 22 Set 2017. 